\title{
Recognition of vehicle make and model in low light conditions
}

\author{
Aymen Fadhil Abbas', Usman Ullah Sheikh², Mohd Norzali Haji Mohd ${ }^{3}$ \\ ${ }^{1,2}$ School of Electrical Engineering, Faculty of Engineering, Universiti Teknologi Malaysia, Malaysia \\ ${ }^{3}$ Faculty of Electrical and Electronics Engineering, Universiti Tun Hussein Onn Malaysia, Malaysia
}

\begin{tabular}{l} 
Article Info \\
\hline Article history: \\
Received Oct 22, 2019 \\
Revised Dec 30, 2019 \\
Accepted Jan 31, 2020 \\
\hline
\end{tabular}

\section{Keywords:}

Image enhancement

Low light

Surveillance systems

Vehicle make and model

recognition

\begin{abstract}
This paper presents a method for vehicle make and model recognition (MMR) in low lighting conditions. While many MMR methods exist in the literature, these methods are designed to be used only in perfect operating conditions. The various camera configuration, lighting condition, and viewpoints cause variations in image quality. In the presented method, the vehicle is first detected, image enhancement is then carried out on the detected front view of the vehicle, followed by features extraction and classification. The performance is then examined on a low-light dataset. The results show around $6 \%$ improvement in the ability of MMR with the use of image enhancement over the same recognition model without image enhancement.
\end{abstract}

This is an open access article under the CC BY-SA license.

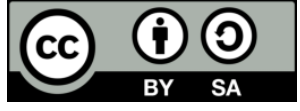

\section{Corresponding Author:}

Usman Ullah Sheikh,

School of Electrical Engineering, Faculty of Engineering,

Universiti Teknologi Malaysia, Johor Bahru, Malaysia.

Email: usman@fke.utm.my

\section{INTRODUCTION}

The use of vehicle make and model recognition (MMR) has applications in surveillance systems and has benefits over using traditional license-plate recognition, which can be easily tricked [1]. This could provide major efficiency improvements for traffic and law enforcement. MMR can also be used with offline and online systems to discover fraudulent license plates by checking the registration database. Major highway traffic can also be monitored by computing the travel time for each vehicle traveling between two cameras [2]. It can also be used in automated vehicle parking systems to further optimize the parking space by the knowledge of MMR [1]. The techniques used in the literature for MMR could be classified into appearance-based, feature-based, and model-based [3-10], and in some cases, a combination of methods is used [11]. Additionally, many classifiers are presented in the literature to improve the detection rate [12]. Regardless of the high accuracy achieved by these recognition methods, there are still several main issues to be addressed [3]. The majority of these methods were designed to work under good lighting conditions, which might not always be the case when a surveillance system is used [13]. Occlusions may also occur when the camera is not able to capture the entire view of the vehicle, causing some vehicle features to be missed which will result in lower recognition accuracy. Moreover, due to the diverse traffic cameras hardware used, images are captured with different resolutions, distortion, limited lighting, visibility and noise which varies between sunny and rainy days and backlight conditions. There is also the issue of viewpoint caused bythe vehicle movement and the limited range of the camera.

This paper presents a new MMR method which employs feature-based recognition techniques, designed specifically for low light conditions. Given a captured image, vehicle detection and alignment are first performed to extract the vehicle from the background using an appearance-based method, then an 
image enhancement technique is applied to normalize the image to normal lighting conditions. With the enhanced image, region-of-interests are identified and vehicle features such as vehicle headlights, grilles and logos are extracted. Then a generic algorithm is utilized to select the finest set of features. Vehicle models are then recognized from these features using a classifier. The proposed system can work on the current implemented traffic surveillance systems with sufficient speed to operate as an online recognition system, and it can be utilized for offline detection. The main contribution of this paper is vehicle recognition in low lighting using an image enhancement technique, particularly,

- this paper improves the detection and alignment process using an appearance-based method, which increases the capability and robustness of the system to be used with all types of camera hardware.

- this paper presents an image enhancement technique to normalize the image to normal lighting conditions.

- this paper proposes a feature-based approach of vehicle make and model recognition and creates a vehicle Image database for performance assessment.

In the following sections, the related works are first introduced, followed by the methodology. The method is then tested against the experimental database, and the performance is examined.

\section{RELATED WORKS}

Recently, many studies have proposed multiple MMR methods. Appearance-based MMR comprises of methods based on dimensions [4], shapes, and textures [5]. These techniques require minimum processing; however, the performance of these techniques varies with the placement and the viewpoint of the camera used. Feature-based MMR techniques are the most widely used and these techniques depend on local or global features of the vehicle such as geographical feature, edge map [6, 7], histogram of gradient, sparse representation, scale-invariant feature transform (SIFT) [8] and symmetrical speeded-up robust features (SURF) $[9,10]$. The performance of these methods depends mainly on training images and the collected features. Model-based MMR [11, 12] could deliver very high accuracy; however, it is inapplicable in time-sensitive applications or online recognition. Additionally, some studies [2, 13] combined multiple features to gain higher recognition accuracy.

While these methods achieved high accuracy, many issues face the performance of these methods in low light or night time where cameras could not clearly capture the appearance of the vehicle and its details. The study in [14] presented a classifier ensemble to address the problem at night by extracting the taillights shape and license plate from the rear view of the vehicle to avoid image distortion generated from the front light beam. Other works classified vehicles by their types at night $[15,16]$; however, did not perform MMR. In a low light condition such as in a cloudy or rainy day, the quality of the image is reduced making it a challenging task to perform MMR.

Images taken in low-light condition are often of low visibility. Consequently, an enhancement to the image is suggested here in order to achieve high accuracy MMR. The use of image enhancement techniques could prepare the image and make it easier to perform object recognition techniques [17, 18]. Currently available image enhancement techniques can be classified into global enhancement [19, 20] and local enhancement [21-23]. Global enhancement equally processes all the pixels in the image regardless of their location resulting in oversaturation and details loss. To overcome these issues, non-linear enhancement techniques could be used such as power-law [24], logarithm [25] and gamma function. Additionally, histogram equalization (HE) could increase the contrast and become a widely-used technique. Local enhancement takes the pixel location into account obtaining better results; however, they might suffer from image hazing or under enhancement, de-hazing techniques are usually used to perform low-light image enhancement [26, 27]. This paper proposes a feature-based method for MMR in low-light conditions by using an image enhancement technique before the feature extraction stage. To preserve the image details and only enhance the image in order to achieve better MMR, in-camera processing details should be taken into account, and the image enhancement technique is designed in the framework of MMR.

\section{METHODOLOGY}

Initially, the vehicle boundaries are extracted from the background by texture analysis. Due to the low-light conditions, this becomes a challenging task. To overcome this, the appearance-based framework by $[28,29]$ is applied. The framework is based on the histogram of oriented gradient (HOG) features, and the HOG features are presented in two scales as shown in Figure 1. General HOG features are detected using a layer covering the entire image. Finer features are detected using a smaller layer that is moved within the image, while the general detection is used as a reference. Initially, the image is divided into smaller pixel regions, and a histogram is created for the local features of each of these regions. The histogram is then 
normalized with the neighboring regions. Rectangular filters are then defining the weights for each detected window on the image, the value of a filter is calculated by the dot product of the weight vector and the features in the detected window on the image. The framework is then trained using actual collected low-light images. The result of this stage, where the vehicle boundaries are extracted is shown in Figure 2. Image enhancement is then carried out on the detected front view of the vehicle, followed by features extraction and classification, as shown in the following sub-sections.

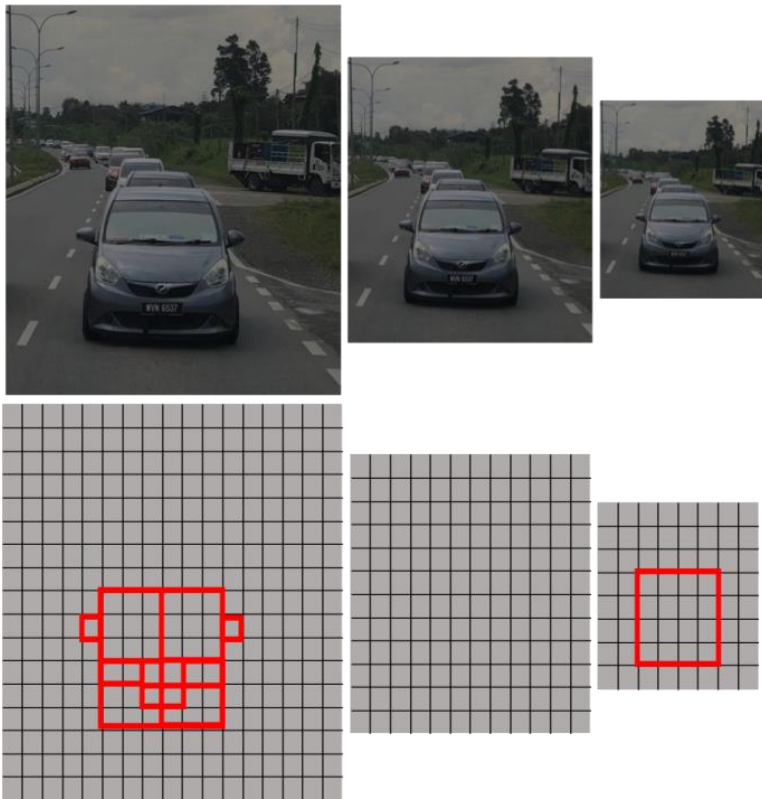

Figure 1. The HOG features, with the placement of the main filter and the part filters



Figure 2. Vehicle boundaries detection by the appearance-based framework

\subsection{Image enhancement}

The multi-exposure fusion framework [30] is used. Normally, the amount of light reaching the camera $\mathbf{E}$ consists of two parts, which are the scene reflectance $\mathbf{R}$ and the illumination map $\mathbf{T}$ and defined as follow:

$$
\mathbf{E}=\mathbf{R} \cdot \mathbf{T}
$$

for most cameras, a response function $f$ is affecting the input image $\mathbf{P}$ and the output image $\mathbf{P}^{\prime}$ such as:

$$
\mathbf{P}=f(\mathbf{E}), \quad \mathbf{P}^{\prime}=f(\mathbf{R})
$$

the output enhanced image could then be written as ;

$$
\begin{aligned}
& \mathbf{P}^{\prime}=f(\mathbf{R})=f\left(\mathbf{E} \cdot \frac{1}{\mathbf{T}}\right) \\
& =g\left(f(\mathbf{E}), \frac{1}{\mathbf{T}}\right)=g\left(\mathbf{P}, \frac{1}{\mathbf{T}}\right)
\end{aligned}
$$

where $g$ is the brightness transform function (BTF), and 1/Tcould be defined here as the exposure ratio.

To measure the optimal exposure ratio, the work here is carried out in two stages as shown in Figure 3, the first is where the adjustment of exposure generates a multi-exposure image set-followed by fusing the generated images into one enhanced result. During the second stage, the illumination estimation method is used to create a weight matrix to derive the camera response model from each exposure. Then the optimal exposure for the camera response model can be found, and the resulted enhanced image is obtained from the original image and the weight matrix. 


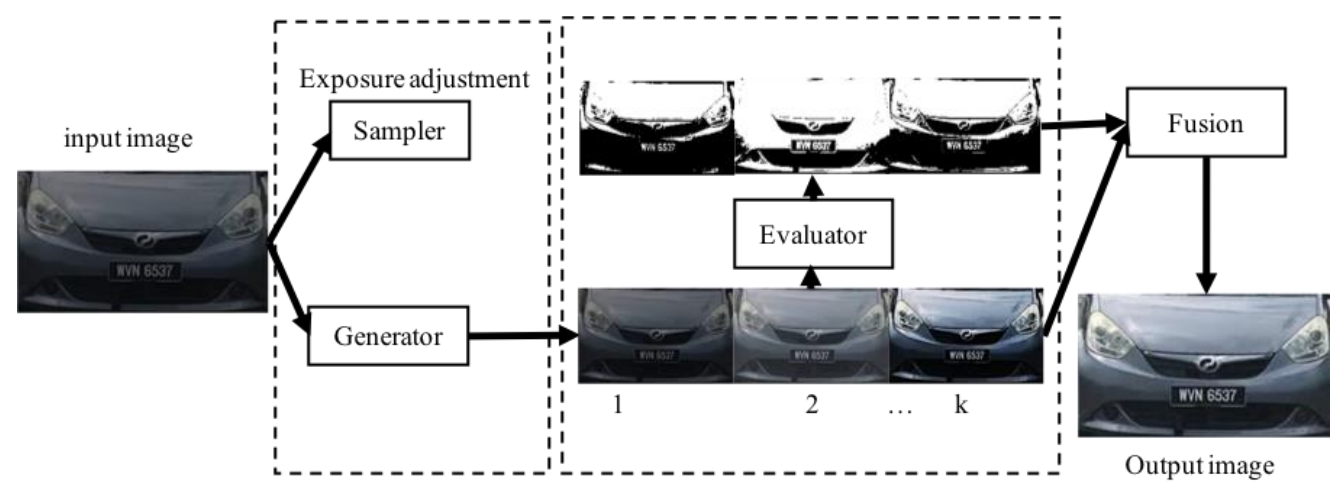

Figure 3. The multi-exposure image enhancement framework

\subsection{Feature set extraction}

The front view of the vehicle is used to extract the features. As shown in Figure 4, the extracted features are the position and shape of the vehicle components such as headlamps, logo, license-plate and the distances between headlamps and license-plate. The detection of license-plate location is done as the first step to set a reference point to find the other vehicle features. This could be done by many methods [1]. Here the technique presented by [31] is used to detect the license-plate. The technique is established by two of the main license plate detection models, which are the candidate extraction as the first stage and the license plate verification process in the second stage. In the first stage, the technique uses edge-based method which is taken from the grayscale images. Then, vertical edges are extracted by applying the Sobel's vertical edge detection algorithm where the license plate has more symmetric vertical edges than the other parts. Following that, mathematical operations are carried out utilizing the license plate structure constraints. Leading to the identification of the license plate candidate regions. Afterward, main candidates are selected by using the license plate aspect ratio. Finally, to verify the position, the standard deviation of the grayscale distribution is applied in the regions and the intensity region with the largest standard deviation is identified as the license plate position. The process was tested in many lighting conditions and showed that license-plates are reliably detectable, and to confirm the license-plate location, the standard deviation of the grey-level distribution was calculated to reassure the position [31]. The headlights are considered as the second reference after the license-plate. Headlights are considered as the most reliable feature in low-lighting conditions. Following the method by [32], the location and shape of the headlights are extracted, where the image has high dense textures in the headlight area and low dense texture on the rest of the hood. Figure 5 shows the result of headlight detection and the contour is highlighted. While the position of license-plate and headlights are reliable enough to determine the vehicle model, the location of auxiliary parts is not consistent, for that these features are not considered as main features. Therefore, to achieve higher classification accuracy, a feature identification technique is applied to optimize the secondary features, which could produce more robust recognition and improve computation time.

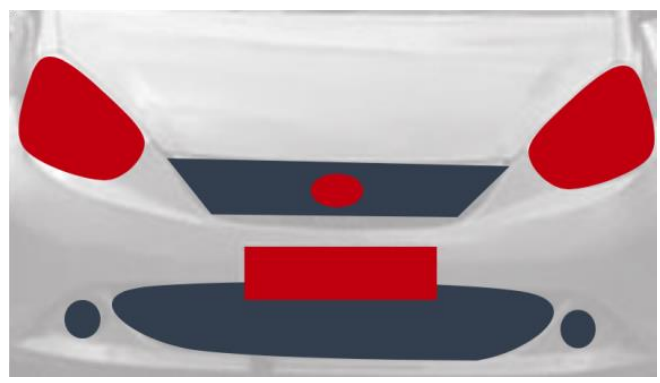

Figure 4. The vehicle features extraction
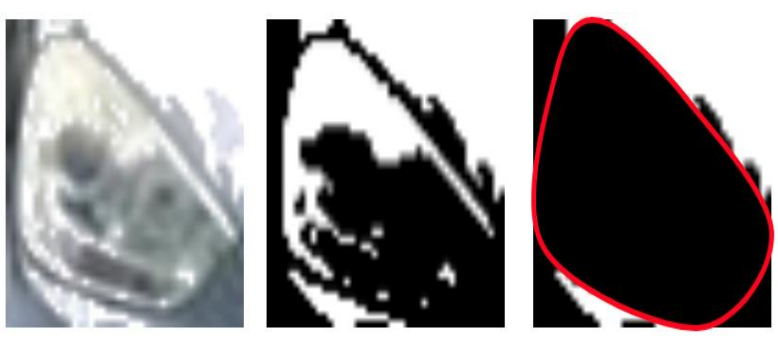

Figure 5. Extraction of headlight contour

\subsection{Classification}

The k-nearest neighbor method (KNN) is used in this study; it was proven that it could achieve higher accuracy [33] in comparison with other classifiers. KNN classifies the test data by comparing 
with kNN training database on a distance function. The Euclidean function was used to measure the distance. The predicted class is defined as:

$$
\begin{aligned}
& \mathrm{NN}=\min _{c \in-1,+1} \operatorname{dist}\left(\sqrt{\left(X_{c}-X_{\text {test }}\right)^{2}}\right) \\
& \mathrm{KNN}=\frac{1}{k} \mathrm{NN}
\end{aligned}
$$

where $c$ is the number of predesigned classes and $k$ is the number of neighbours, $X_{c}$ and $X_{\text {test }}$ are predefined classes and test features. The majority vote for three, five and seven nearest neighbors were used in this study to reduce the variance and highlight the final prediction.

\section{EXPERIMENTAL RESULT}

The accuracy of the proposed method was examined in different scenarios, with and without the image enhancement technique to investigate the capability of the enhancement on different image quality and exposure. Further, a comparison with the methods used in other works is done to assess the performance of the proposed method. The test database contains 2,191 vehicle images, given in Table 1, and examples of vehicle images can be seen in Figure 6. Images are selected to cover a broad range of viewpoints and light conditions. The majority of the vehicles were picked based on their popularity in the observation area.

The confusion matrix of the proposed approach is shown in Figure 7. The test is done using the data set of each model versus all models. Only a few missed recognitions for some of the models were observed, which might be due to the limited lighting, and the similarity between these models. The overall accuracy of the method is $97.31 \%$. To evaluate the use of image enhancement technique, the classification accuracy is examined with and without the image enhancement. Additionally, it's compared with other methods which are not designed for low light conditions. The performance of the method on the test dataset is reported in Table 2.

Table 1. Vehicle models dataset

\begin{tabular}{lcclcc}
\hline \multicolumn{1}{c}{ Make and Model } & No. of Images & Years of the model & Make and Model & No. of Images & Years of the Model \\
\hline Ford Escape & 60 & 1 & Nissan Sentra & 54 & 2 \\
Ford Mondeo & 76 & 4 & Nissan Tena & 56 & 1 \\
Ford Tierra & 34 & 1 & Nissan Tilda & 112 & 1 \\
Honda City & 55 & 4 & PeroduaAxia & 24 & 1 \\
Honda Civic & 69 & 1 & PeroduaMyvi & 84 & 4 \\
Honda CRV & 204 & 4 & Proton Persona & 68 & 4 \\
Honda Fit & 55 & 2 & Proton Saga & 146 & 4 \\
Mitsubishi Lancer & 16 & 1 & Proton Waja & 50 & 4 \\
Mitsubishi Outlander & 27 & 1 & Toyota Previa & 22 & 1 \\
Mitsubishi Savrin & 41 & 1 & Toyota Rav & 73 & 1 \\
Nissan Cefiro & 114 & 2 & Toyota Surf & 40 & 1 \\
Nissan Livna & 122 & 1 & Toyota Tercel & 109 & 4 \\
Nissan March & 80 & 2 & Toyota Vios & 96 & 4 \\
\hline
\end{tabular}
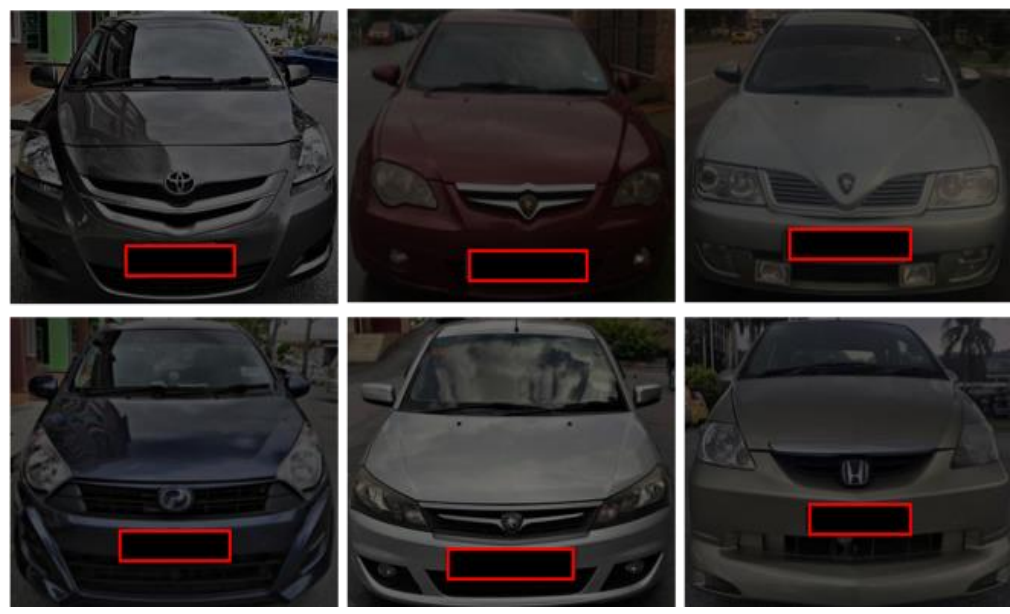

Figure 6. Sample images from the vehicle database 


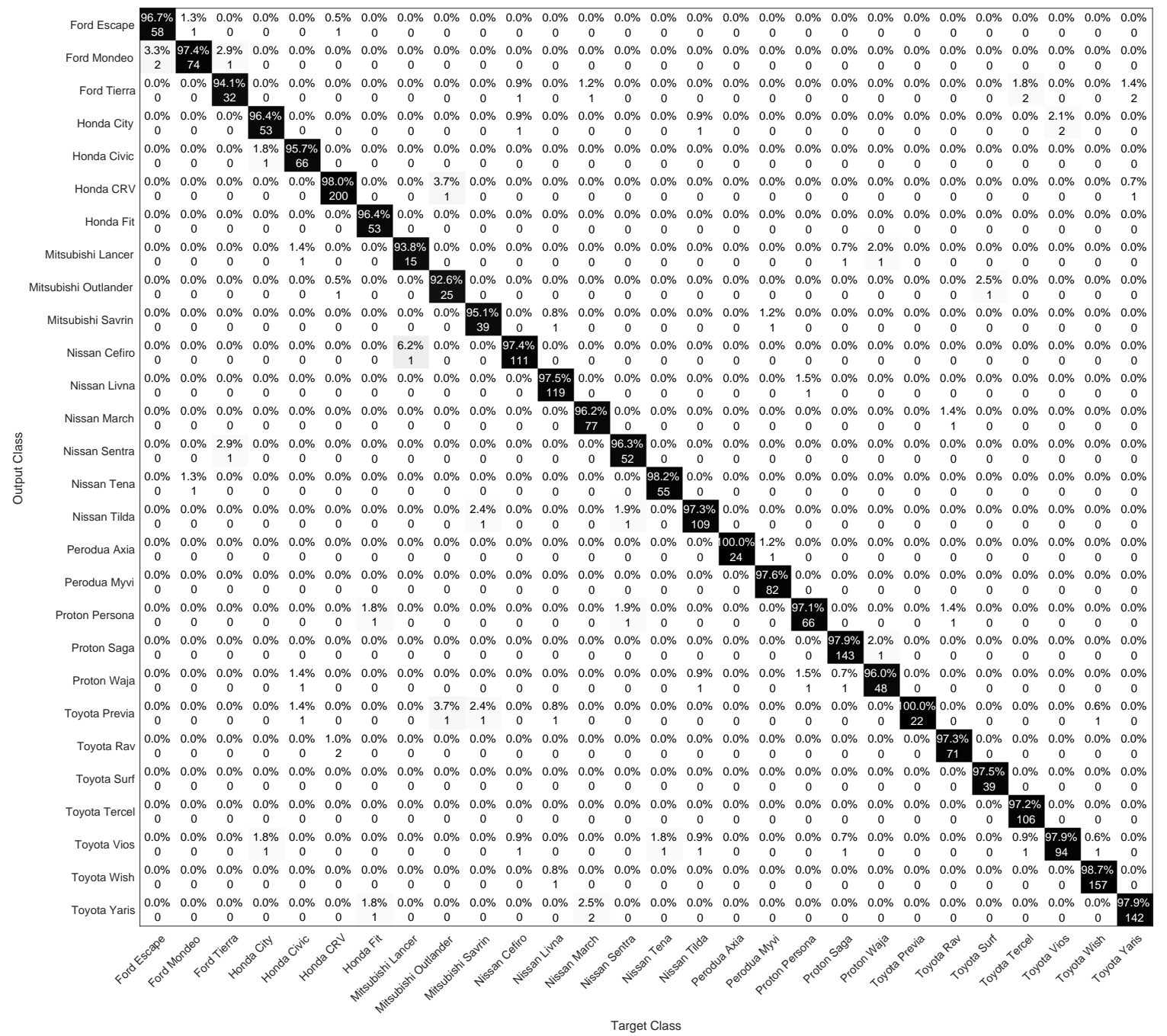

Figure 7. The confusion matrix for the proposed approach with an overall accuracy of $97.31 \%$

Table 2. Performance comparison with and without image enhancement

\begin{tabular}{cccc}
\hline Method & Description & Accuracy \\
\hline $\begin{array}{c}\text { The proposed method } \\
\text { (With image enhancement) }\end{array}$ & The presented method, with the use of image enhancement prior to & the feature extraction stage \\
The proposed method & The same presented criteria, without the use of image enhancement & $97.31 \%$ \\
(Without image enhancement) & The method uses classifier ensemble to address the problem at night & $93.80 \%$ \\
Classifier ensemble [14] & The method uses multi-class SVM and structural verification & $91.70 \%$ \\
SIFT, SURF, edge histogram [15] & The &
\end{tabular}

\section{CONCLUSION}

This paper proposed the use of image enhancement technique to improve the recognition in low light situations. Noticeable improvement of around 6\% using image enhancement is obtained in the test data versus the same system performance without the image enhancement stage. The proposed method showed promising improvement in recognition accuracy. However, there is still ample room for improvement in the vehicle make and model recognition systems.

\section{ACKNOWLEDGEMENTS}

The authors thank the Ministry of Education Malaysia, Universiti Tun Hussein Onn (UTHM) for their support under the Fundamental Research Grant Scheme (FRGS), grant number 1580 and UniversitiTeknologi Malaysia under Research University Grant (GUP), grant number 19H61. 


\section{REFERENCES}

[1] S. Du, M. Ibrahim, M. Shehata and W. Badawy, "Automatic License Plate Recognition (ALPR): A State-of-the-Art Review," in IEEE Transactions on Circuits and Systems for Video Technology, vol. 23, no. 2, pp. 311-325, Feb. 2013.

[2] M. M. Arzani and M. Jamzad, "Car type recognition in highways based on wavelet and contourlet feature extraction," in Proceedings of the 2010 International Conference on Signal and Image Processing, ICSIP 2010, pp. 353-356, 2010.

[3] T. D. Räty, "Survey on Contemporary Remote Surveillance Systems for Public Safety," in IEEE Transactions on Systems, Man, and Cybernetics, Part C (Applications and Reviews), vol. 40, no. 5, pp. 493-515, Sept. 2010.

[4] C. Hoffman, T. Dang and C. Stiller, "Vehicle detection fusing 2D visual features," IEEE Intelligent Vehicles Symposium, 2004, Parma, Italy, pp. 280-285, 2004.

[5] M. Betke, E. Haritaoglu, and L. S. Davis, "Real-time multiple vehicle detection and tracking from a moving vehicle," Mach. Vis. Appl., vol. 12, no. 2, pp. 69-83, 2000.

[6] G. Pearce and N. Pears, "Automatic make and model recognition from frontal images of cars," in 2011 8th IEEE International Conference on Advanced Video and Signal Based Surveillance, AVSS 2011, pp. 373-378, 2011.

[7] D. T. Munroe and M. G. Madden, "Multi-class and single-class classification approaches to vehicle model recognition from images," in 16th Irish Conference on Artificial Intelligence and Cognitive Science, pp. 93-102, 2005.

[8] A. Psyllos, C. N. Anagnostopoulos, and E. Kayafas, "Vehicle model recognition from frontal view image measurements," in Computer Standards and Interfaces, vol. 33, no. 2, pp. 142-151, 2011.

[9] R. Baran, A. Glowacz, and A. Matiolanski, "The efficient real- and non-real-time make and model recognition of cars," Multimed. Tools Appl., vol. 74, no. 12, pp. 4269-4288, 2015.

[10] L. C. Chen, J. W. Hsieh, Y. Yan, and D. Y. Chen, "Vehicle make and model recognition using sparse representation and symmetrical SURFs," Pattern Recognit., vol. 48, no. 6, pp. 1979-1998, 2015.

[11] M. J. Leotta and J. L. Mundy, "Vehicle Surveillance with a Generic, Adaptive, 3D Vehicle Model," in IEEE Transactions on Pattern Analysis and Machine Intelligence, vol. 33, no. 7, pp. 1457-1469, July 2011.

[12] D. Roller, K. Daniilidis, and H. H. Nagel, "Model-based object tracking in monocular image sequences of road traffic scenes," Int. J. Comput. Vis., vol. 10, no. 3, pp. 257-281, 1993.

[13] B. Zhang, "Reliable Classification of Vehicle Types Based on Cascade Classifier Ensembles," in IEEE Transactions on Intelligent Transportation Systems, vol. 14, no. 1, pp. 322-332, March 2013.

[14] N. Boonsim and S. Prakoonwit, "Car make and model recognition under limited lighting conditions at night," Pattern Anal. Appl., vol. 20, no. 4, pp. 1195-1207, 2017.

[15] Chun-Che Wang, Shih-Shinh Huang and Li-Chen Fu, "Driver assistance system for lane detection and vehicle recognition with night vision," 2005 IEEE/RSJ International Conference on Intelligent Robots and Systems, Edmonton, Alta, pp. 3530-3535, 2005.

[16] S. Görmer, D. Müller, S. Hold, M. Meuter, and A. Kummert, "Vehicle recognition and TTC estimation at night based on spotlight pairing," in IEEE Conference on Intelligent Transportation Systems, Proceedings, ITSC, pp. 196-201, 2009.

[17] S. Wang, J. Zheng, H. Hu and B. Li, "Naturalness Preserved Enhancement Algorithm for Non-Uniform Illumination Images," in IEEE Transactions on Image Processing, vol. 22, no. 9, pp. 3538-3548, Sept. 2013.

[18] H. Ibrahim and N. S. Pik Kong, "Brightness Preserving Dynamic Histogram Equalization for Image Contrast Enhancement," in IEEE Transactions on Consumer Electronics, vol. 53, no. 4, pp. 1752-1758, Nov. 2007.

[19] A. M. Reza, "Realization of the contrast limited adaptive histogram equalization (CLAHE) for real-time image enhancement," J. VLSI Signal Process. Syst. Signal Image. Video Technol., vol. 38, no. 1, pp. 35-44, 2004.

[20] K. S. Sim, C. P. Tso, and Y. Y. Tan, "Recursive sub-image histogram equalization applied to gray scale images," Pattern Recognit. Lett., vol. 28, no. 10, pp. 1209-1221, 2007.

[21] L. Wang, L. Xiao, H. Liu and Z. Wei, "Variational Bayesian Method for Retinex," in IEEE Transactions on Image Processing, vol. 23, no. 8, pp. 3381-3396, Aug. 2014.

[22] X. Fu, D. Zeng, Y. Huang, X. Zhang and X. Ding, "A Weighted Variational Model for Simultaneous Reflectance and Illumination Estimation," 2016 IEEE Conference on Computer Vision and Pattern Recognition (CVPR), Las Vegas, NV, pp. 2782-2790, 2016.

[23] X. Guo, Y. Li and H. Ling, "LIME: Low-Light Image Enhancement via Illumination Map Estimation," in IEEE Transactions on Image Processing, vol. 26, no. 2, pp. 982-993, Feb. 2017.

[24] A. Beghdadi and A. Le Negrate, "Contrast enhancement technique based on local detection of edges," Comput. Vision, Graph. Image Process., vol. 46, no. 2, pp. 162-174, 1989

[25] E. Peli, "Contrast in complex images.," J. Opt. Soc. Am. A., vol. 7, no. 10, pp. 2032-40, 1990.

[26] L. Li, R. Wang, W. Wang and W. Gao, "A low-light image enhancement method for both denoising and contrast enlarging," 2015 IEEE International Conference on Image Processing (ICIP), Quebec City, QC, pp. 3730-3734, 2015.

[27] Xuan Dong et al., "Fast efficient algorithm for enhancement of low lighting video," 2011 IEEE International Conference on Multimedia and Expo, Barcelona, pp. 1-6, 2011.

[28] P. Felzenszwalb, D. McAllester, and D. Ramanan, "A discriminatively trained, multiscale, deformable part model," in 26th IEEE Conference on Computer Vision and Pattern Recognition, CVPR, pp. 115-129, 2008.

[29] D. Forsyth, "Object detection with discriminatively trained part-based models," Computer (Long. Beach. Calif)., vol. 47, no. 2, pp. 6-7, 2014. 
[30] Z. Ying, G. Li, Y. Ren, R. Wang and W. Wang, "A New Low-Light Image Enhancement Algorithm Using Camera Response Model," 2017 IEEE International Conference on Computer Vision Workshops (ICCVW), Venice, pp. 3015-3022, 2017.

[31] P. R. Mendes Júnior, J. M. R. Neves, A. I. Tavares and D. Menotti, "Towards an automatic vehicle access control system: License plate location," 2011 IEEE International Conference on Systems, Man, and Cybernetics, Anchorage, AK, pp. 2916-2921, 2011.

[32] N. Boonsim and S. Prakoonwit, "An Algorithm for Accurate Taillight Detection at Night," Int. J. Comput. Appl., vol. 100, no. 12, pp. 31-35, 2014.

[33] D. R. Amancio et al., "A systematic comparison of supervised classifiers," PLoS One, vol. 9, no. 4, pp. 300-320, 2014.

\section{BIOGRAPHIES OF AUTHORS}

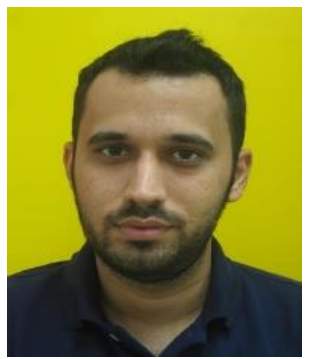

Aymen Fadhil Abbas completed his bachelor degree in 2011 from University of Technology, Baghdad, Iraq. Received his M.Sc. Degree from UniversitiTeknologi Malaysia, Johor Bahru, Malaysia in 2014. He is currently working toward the Ph.D. degree in computer and microelectronic systems with UniversitiTeknologi Malaysia. His research interests include intelligent surveillance systems, computer vision, intelligent transportation systems and vehicle make and model recognition.

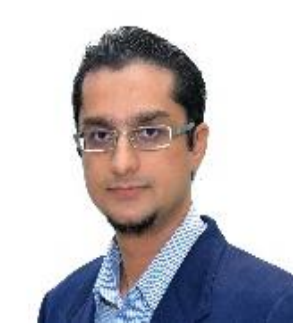

Usman Ullah Sheikh received his Ph.D. degree (2009) in image processing and computer vision from UniversitiTeknologi Malaysia. His research work is mainly on computer vision, machine learning, and embedded systems design. He is currently a Senior Lecturer at UniversitiTeknologi Malaysia.



Mohd Norzali Haji Mohd is currently working as Senior Lecturer, Faculty Lab Manager (HoD) and Former Industrial Training Coordinator at the Department of Computer Engineering, Faculty of Electrical and Electronic Engineering, Universiti Tun Hussein Onn Malaysia (UTHM). He received B.Eng., M.Eng. from Fukui University, Japan in 2002 and 2004 respectively. In April 2015, he received Ph.D. from the Department of Information Sciences and Biomedical Engineering, Kagoshima University, Japan. His research interests are computer vision, robot vision, pattern recognition, and image processing. 